\title{
Fully Nonstationary Spatially Variable Ground Motion Simulations Based on a Time-Varying Power Spectrum Model
}

\author{
Huiguo Chen, ${ }^{1,2}$ Yingmin Li, $^{1}$ and Junru Ren ${ }^{2}$ \\ ${ }^{1}$ College of Civil Engineering, Chongqing University, Chongqing 400045, China \\ ${ }^{2}$ Department of Civil Engineering, Logistical Engineering University, Chongqing 401311, China \\ Correspondence should be addressed to Huiguo Chen; chenhg_mail@163.com
}

Received 9 March 2014; Revised 21 July 2014; Accepted 23 July 2014; Published 27 August 2014

Academic Editor: Carsten Proppe

Copyright (c) 2014 Huiguo Chen et al. This is an open access article distributed under the Creative Commons Attribution License, which permits unrestricted use, distribution, and reproduction in any medium, provided the original work is properly cited.

\begin{abstract}
By analyzing the evolutionary spectrum method for multivariate nonstationary stochastic processes, a simulation method for fully nonstationary spatially variable ground motion is proposed based on the Kameda time-varying power spectrum model. This method can properly simulate nonstationary spatially variable ground motion based on a target response spectrum. Two numerical examples, in which the Kameda time-varying power spectra are calculated for different conditions, are presented to demonstrate the capabilities of the proposed method. In the first example, the nonstationary spatially variable ground motion that satisfies the timefrequency characteristics and response characteristics of the original ground motion is simulated by identifying the parameters of the given time-varying power spectrum. In the second example, the ground motion that satisfies the design response spectra is simulated by defining the parameters of the time-varying power spectrum directly. The results demonstrate that the method can effectively simulate nonstationary spatially variable ground motion, which implies that the proposed method can be used in engineering applications.
\end{abstract}

\section{Introduction}

The spatial variability of earthquake ground motion can have significant effects on the response of extended structures, such as long bridges, tunnels, and life-line systems. It is unrealistic to analyze the dynamic seismic response based solely on the actual ground motion records because the seismic array record is unrepeatable in practice and extended structures typically have variable spatial characteristics. In this case, the simulation of artificial spatially variable ground motion is required; such simulation results can be used as inputs to the deterministic seismic response analysis of structural supports.

The Monte Carlo simulation technique is the most common method of ground motion simulation; however, it is difficult to accurately simulate ground motions that are nonstationary in both the time and the frequency domains because it is difficult to describe the nonstationary characteristics and define the sampling functions and filtering parameters. Evolutionary stochastic process theory [1] (Priestley. 1967) transforms a nonstationary process into an integration of a stationary process and a deterministic modulation function, which allows the time-varying spectrum density of the nonstationary process to be derived from the spectrum density of the stationary process. Compared with the traditional Monte Carlo method, this theory is more efficient in describing the frequency variation of nonstationary processes. Researchers have used this theory to establish a variety of spectrum representation methods and nonstationary ground motion models for different nonstationary processes [1-3]. Shinozuka [4] first applied evolutionary stochastic process theory to simulate multidimensional and spatially variable nonstationary ground motions. Many researchers, including Deodatis [5], Conte and Peng [6], Yang [7], Grigoriu [8], and Ancheta [9], have further developed this method. This method is widely accepted by engineers because it considers spatial changes in ground motion correlation at every point and is able to match the target power spectra as well as possible. However, a limitation of the spectrum representation method is that its time-varying power spectrum is difficult to model in practice. Thus, a simplified non-time-varying power spectrum model is typically employed. This deficiency can degenerate 
the ground motion simulation to a nonstationary time pattern instead of a nonstationary time-frequency pattern.

To solve the nonstationary frequency problem in ground motion simulations, Deodatis [2] proposed a variation of the time-varying power spectrum model based on the analysis of site liquefaction properties of the 1964 Niigata ground motion. However, the model only uses the time-frequency characteristics of the Niigata ground motion and is unable to simulate ground motions with different nonstationary timefrequency characteristics; therefore, it is difficult to apply it to other situations. Using the multifiltering technique, Kameda [10] established a time-varying power spectrum model that allows for the simulation of nonstationary ground motion. Many researchers have investigated the parameter selection and attenuating behavior of this model. However, the model is mainly applied to simulate single-point ground motion and has not been applied to spatially variable ground motion simulations.

By analyzing the evolutionary spectrum method for nonstationary stochastic processes, this paper proposes a nonstationary spatially variable ground motion simulation method based on the Kameda time-varying power spectrum model. This method can reflect the nonstationary time-frequency characteristics and fit the target response spectrum well under the conditions of the given spatial characteristics. Two numerical examples are presented to verify the capabilities of the proposed method. The first example focuses on simulating the time-frequency characteristics of the actual ground motion, whereas the second focuses on the response spectra of the design code.

\section{Evolutionary Spectrum Method for Nonstationary Stochastic Processes}

According to the Priestley nonstationary stochastic model, which is based on the evolutionary spectrum density [1], a nonstationary stochastic process with an average value of zero can be expressed as

$$
y(t)=\int_{-\infty}^{+\infty} A(\omega, t) e^{i \omega t} d \mathbf{F}_{x}(\omega),
$$

where $\mathbf{F}_{x}(\omega)$ is an orthogonal complex stationary stochastic process and $A(\omega, t)$ is a modulating function that is subject to the conditions outlined by Priestley [1].

Assume one stationary process that satisfies

$$
x(t)=\int_{-\infty}^{\infty} e^{i \omega t} d \mathbf{F}_{x}(\omega) .
$$

The evolutionary spectrum (or time-varying power spectrum) of $y(t)$ can be expressed as

$$
S_{y}(\omega, t)=|A(\omega, t)|^{2} S_{x}(\omega),
$$

where $S_{x}(\omega)$ is the power spectral density function of $x(t)$.

According to the spectral representation method recommended by Tsai and Hashash [3], we assume the average values of the components $y_{1}(t), y_{2}(t), \ldots, y_{m}(t)$ in a nonstationary stochastic process to be equal to zero:

$$
E\left[y_{j}(t)\right]=0, \quad(j=1, \ldots, m) .
$$

The cross-correlation matrix is given by

$$
\begin{aligned}
\mathbf{R}_{y}(t, t+\tau) & \\
= & {\left[\begin{array}{cccc}
R_{11}(t, t+\tau) & R_{12}(t, t+\tau) & \cdots & R_{1 m}(t, t+\tau) \\
R_{21}(t, t+\tau) & R_{22}(t, t+\tau) & \cdots & R_{2 m}(t, t+\tau) \\
\vdots & \vdots & \ddots & \vdots \\
R_{m 1}(t, t+\tau) & R_{m 2}(t, t+\tau) & \cdots & R_{m m}(t, t+\tau)
\end{array}\right] }
\end{aligned}
$$

and the corresponding cross-spectral density matrix is

$$
\mathbf{S}_{y}(\omega, t)=\left[\begin{array}{cccc}
S_{11}(\omega, t) & S_{12}(\omega, t) & \cdots & S_{1 m}(\omega, t) \\
S_{21}(\omega, t) & S_{22}(\omega, t) & \cdots & S_{2 m}(\omega, t) \\
\vdots & \vdots & \ddots & \vdots \\
S_{m 1}(\omega, t) & S_{m 2}(\omega, t) & \cdots & S_{m m}(\omega, t)
\end{array}\right] .
$$

The elements of the cross-spectral density matrix are defined as

$$
\begin{gathered}
S_{j j}(\omega, t)=\left|A_{j}(\omega, t)\right|^{2} S_{j}(\omega), \quad j=1,2, \ldots, m \\
S_{j k}(\omega, t)=\sqrt{S_{j j}(\omega, t) S_{k k}(\omega, t)} \Gamma_{j k}(\omega) \\
j=1,2, \ldots, m ; j \neq k,
\end{gathered}
$$

where $S_{j}(\omega)$ is the stationary power spectral density function of $y_{j}(t),(j=1, \ldots, m)$ and $\Gamma_{j k}(\omega)$ is the complex coherence function between $y_{j}(t)$ and $y_{k}(t)$.

The cross-power spectral density matrix of the spatial stochastic process $y_{j}(t)$ is Hermitian and positive definite. Therefore, it can be decomposed into the following product according to the Cholesky method:

$$
\mathbf{S}_{y}(\omega, t)=\mathbf{H}(\omega, t) \mathbf{H}^{*}(\omega, t),
$$

where $\mathbf{H}(\omega, t)$ is a lower triangular matrix and the superscript * denotes the transpose of a matrix. $\mathbf{H}(\omega, t)$ is written as

$$
\mathbf{H}(\omega, t)=\left[\begin{array}{cccc}
H_{11}(\omega, t) & 0 & \cdots & 0 \\
H_{21}(\omega, t) & H_{22}(\omega, t) & \cdots & 0 \\
\vdots & \vdots & \ddots & \vdots \\
H_{m 1}(\omega, t) & H_{m 2}(\omega, t) & \cdots & H_{m m}(\omega, t)
\end{array}\right] .
$$

Thus, the spatially stochastic process $y_{j}(t)$ can be simulated as

$$
\begin{array}{r}
y_{j}(t)=2 \sum_{k=1}^{m} \sum_{i=1}^{N}\left|H_{j k}\left(\omega_{i}, t\right)\right| \sqrt{\Delta \omega} \cos \left[\omega_{i} t-\theta_{j k}\left(\omega_{i}, t\right)+\varphi_{k i}\right], \\
j=1,2, \ldots, m,
\end{array}
$$

where

$$
\theta_{j k}\left(\omega_{i}, t\right)=\tan ^{-1}\left(\frac{\operatorname{Im}\left[H_{j k}\left(\omega_{i}, t\right)\right]}{\operatorname{Re}\left[H_{j k}\left(\omega_{i}, t\right)\right]}\right),
$$


where Im and Re denote the imaginary and real parts of a complex number, respectively, $\varphi_{k i}$ is a random phase that is distributed uniformly in the region of $[0,2 \pi], \omega_{i}=i \Delta \omega, i=$ $1,2, \ldots, N, \Delta \omega=\omega_{c} / N, \omega_{c}$ is the upper cutoff frequency, and $N$ is the number of subsection points of the frequency.

Based on the evolutionary power spectrum theory, the specific nonstationary time-frequency spatially variable ground motion can be simulated as long as the time-varying power spectrum function $S_{j j}(\omega, t)$ and the cross-power spectrum function $S_{j k}(\omega, t)$ are defined. Equation (3) shows that the time-varying power spectrum of the ground motion is represented by the product of a stationary autopower spectrum function $S_{x}(\omega)$ and a modulating function $A(\omega, t)$. Thus, the study of the time-varying power spectrum can be converted into the study of the power spectrum and the modulating function. Several theories related to the stationary autopower spectrum function have been presented, such as the K-T spectrum (Kanai, 1957 [12] and Tajimi, 1960 [13]) and the C-P spectrum (Clough and Penzien [14]). Because the autopower spectrum function $S_{x}(\omega)$ is not time dependent, the modulating function $A(\omega, t)$ (also called the nonuniformly modulated function) must be related to time and frequency to create a time-varying power spectrum function. However, the nonuniformly modulated function $A(\omega, t)$ is difficult to model in practice, so it is often simplified to the uniformly modulated function $A(t)$, which is independent of the frequency, such as in the models of Shinozuka and Sato [15] and Amin and Ang [16]. The result of this simplification is that the power spectrum, defined in (3), will lose its timevarying characteristics and the simulated ground motion will no longer be nonstationary in the frequency domain.

\section{Kameda Time-Varying Power Spectrum Model}

Kameda [10] analyzed the distribution of the time-varying power spectra of actual ground motions using the multifiltering technique, proposed a modeling method for the power spectrum curve of each discrete frequency point, and established a uniform presentation for the power spectrum curve of each discrete frequency point. Hence, a time-varying power spectrum can be modeled by numerical fitting. This model can be represented as follows:

$$
\sqrt{G(2 \pi f, t)}=\alpha_{m}(f) \frac{t-t_{s}(f)}{t_{p}(f)} \exp \left(1-\frac{t-t_{s}(f)}{t_{p}(f)}\right),
$$

where $G(2 \pi f, t)$ is the time-varying power spectrum model of ground motion $(f=\omega / 2 \pi), t_{s}(f)$ and $t_{p}(f)$ are the starting time and duration parameters, respectively, and $\alpha_{m}(f)$ is the intensity parameter that represents the peak value of $\sqrt{G\left(2 \pi f_{i}, t\right)}$ for time $t$ and frequency $f_{i}$. These parameters are determined from recorded acceleration time histories.

Figure 1 shows example of recorded and modeled evolutionary power spectra at an assumed frequency point $f_{i}$. The blue line represents the time-varying power spectrum of the actual ground motion at $f_{i}$, and the red line represents the identified time-varying power spectrum calculated using (12). According to the modeling method, the values of $t_{s}(f)$,

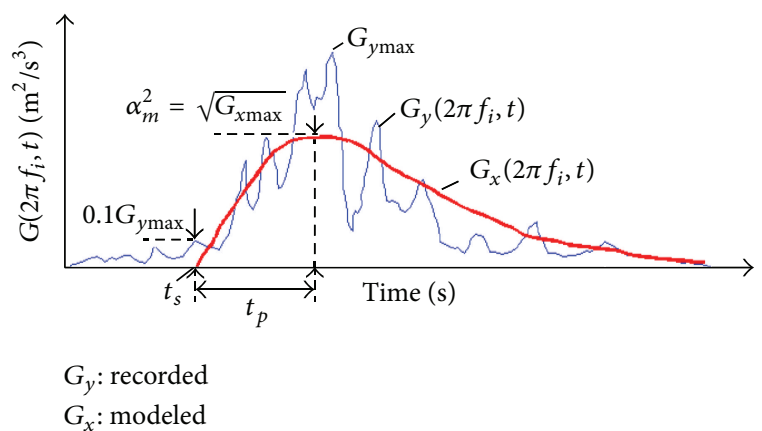

FIgURE 1: Recorded and simulated evolutionary power spectra [11].

$t_{p}(f)$, and $\alpha_{m}(f)$ that correspond to all frequency points can be obtained if the time-varying power spectra at each frequency point are modeled. Thus, we can establish the fully time-varying modeled power spectrum that reflects the nonstationary characteristics of the actual ground motion.

Defining the parameters of the Kameda model by calculating the time-varying power spectrum of a given acceleration is important for simulating the nonstationary time-frequency characteristics of a given ground motion. Therefore, according to the classical evolutionary spectrum method, the first step is to model the time-varying power spectrum of the actual ground motion. When utilizing the Kameda model to model the time-varying power spectrum of the actual ground motion, it is important to obtain an accurate actual timevarying power spectrum and define the appropriate discrete frequency interval to generate accurate model parameters. The HHT method [17], which is suitable for analyzing and processing nonstationary signals, is used to improve the accuracy of the estimated time-varying power spectrum of the ground motion. In defining the discrete frequency interval, a smaller frequency interval allows for a more precise fit. However, a small interval also increases the computational burden of the problem. According to Berrill and Hanks' [18] research on ground motion records, it is appropriate to set the low-frequency cutoff at $0.125 \mathrm{~Hz}$; then, the interval of discrete frequency points is set to $0.125 \mathrm{~Hz}$ to guarantee that the interval satisfies the low-frequency cutoff frequency. The least-squares error method is typically applied to identify the parameters of the Kameda model. The specific procedures used to identify the parameters are as follows.

(1) Calculate the time-varying power spectrum of the known ground motion using the HHT method.

(2) Define $t_{s}\left(f_{i}\right)$ by the peak value of the time-varying power spectrum of each discrete frequency point.

(3) Construct the objective function based on the leastsquares error, as in (13), and define the values of $\alpha_{m}\left(f_{i}\right)$ and $t_{p}\left(f_{i}\right)$ for each discrete frequency point using the optimization method. Consider

$$
F_{\min }=\sum_{i=1}^{m}\left[S\left(2 \pi f_{i}, t\right)-G\left(2 \pi f_{i}, t\right)\right]^{2},
$$




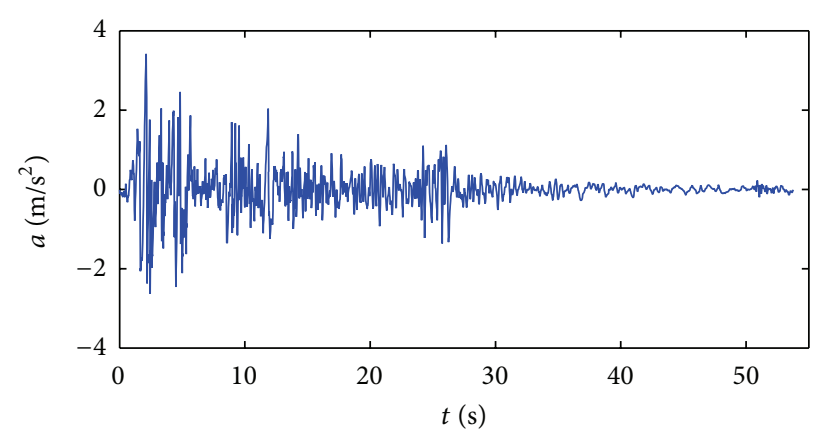

(a) Acceleration

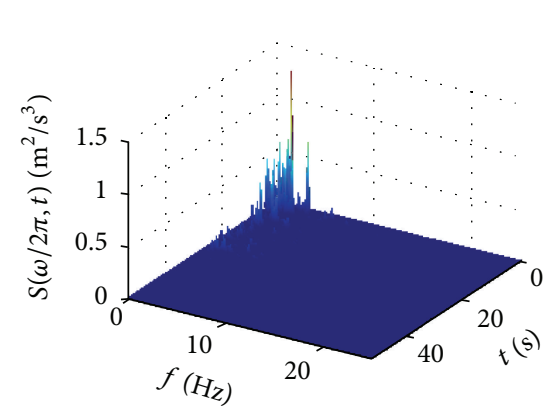

(b) Time-varying power spectrum

FIGURE 2: Acceleration and time-varying power spectrum of the El Centro ground motion record.
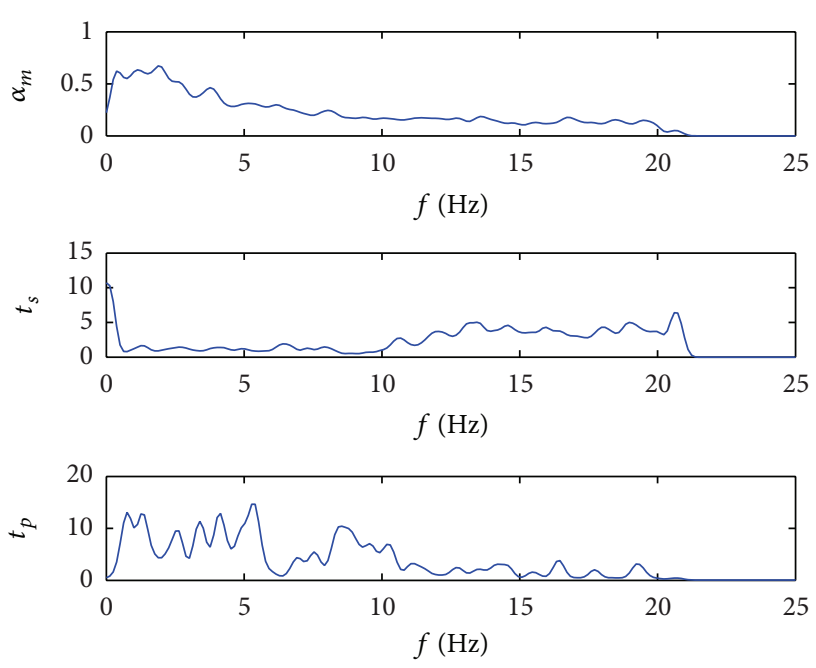

FIGURE 3: Changes in the time-varying power spectrum parameters with frequency for the El Centro ground motion.

where $i$ is the number of discrete frequency points, $S\left(2 \pi f_{i}, t\right)$ is the actual time-varying power spectrum of each frequency point, and $G\left(2 \pi f_{i}, t\right)$ is the modeled time-varying power spectrum of each frequency point.

The El Centro record is used to further explain the process of identifying the parameters of the Kameda time-varying power spectrum model. Figure 2(a) shows the acceleration of the El Centro record, and Figure 2(b) shows its time-varying power spectrum calculated using the HHT method. Figure 3 shows how the identified parameters $\alpha_{m}(f), t_{s}(f)$, and $t_{p}(f)$ of the Kameda model vary with frequency, and Figure 4 shows the modeled time-varying power spectrum calculated with the identified parameters and (12). A comparison of Figures 4 and 2(b) shows that the modeled time-varying power spectrum can reflect the main differences between the actual time-varying power spectrum and the simulated spectrum. To directly evaluate whether the identified parameters $\alpha_{m}\left(f_{i}\right), t_{s}\left(f_{i}\right)$, and $t_{p}\left(f_{i}\right)$ of the Kameda model at each discrete frequency point match the actual time-varying power spectrum of the given ground motion, Figure 5 compares the actual power spectrum and the modeled spectrum at several

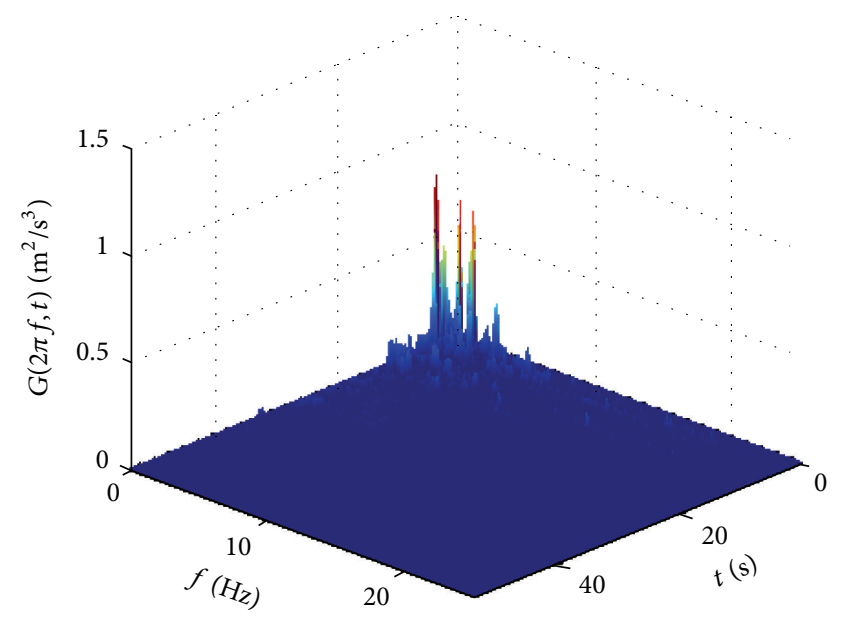

Figure 4: Time-varying power spectrum model of the El Centro ground motion.

typical frequency points. The modeled time-varying power spectrum accurately describes the distribution and peak characteristics of the actual time-varying power spectrum, which demonstrates the ability of the Kameda model to model the given time-varying power spectrum.

In the existing simulation methods, instead of utilizing the known ground motion as the initial sample, basic parameters, such as magnitude, epicentral distance, and site characteristics, can be used to simulate the ground motion. If the characteristics of the time-varying power spectrum must be considered, it is essential to establish the attenuation law of the Kameda model, which varies with these ground motion parameters. To apply the Kameda model to engineering practice, Sugito et al. [11] proposed a typical time-varying power spectrum attenuation law based on the statistical analysis of 118 Japanese ground motion records, as shown in (14) and (15):

$$
\begin{gathered}
\log \alpha_{m}(f)=B_{0}(f)+B_{1}(f) M-B_{2}(f) \log (R) \\
\log t_{p}(f)=P_{0}(f)+P_{1}(f) M-P_{2}(f) \log (R) \\
\hat{t}_{s}(f)=t_{s}(f)-t_{m}=S_{0}(f)+S_{1}(f) M,
\end{gathered}
$$



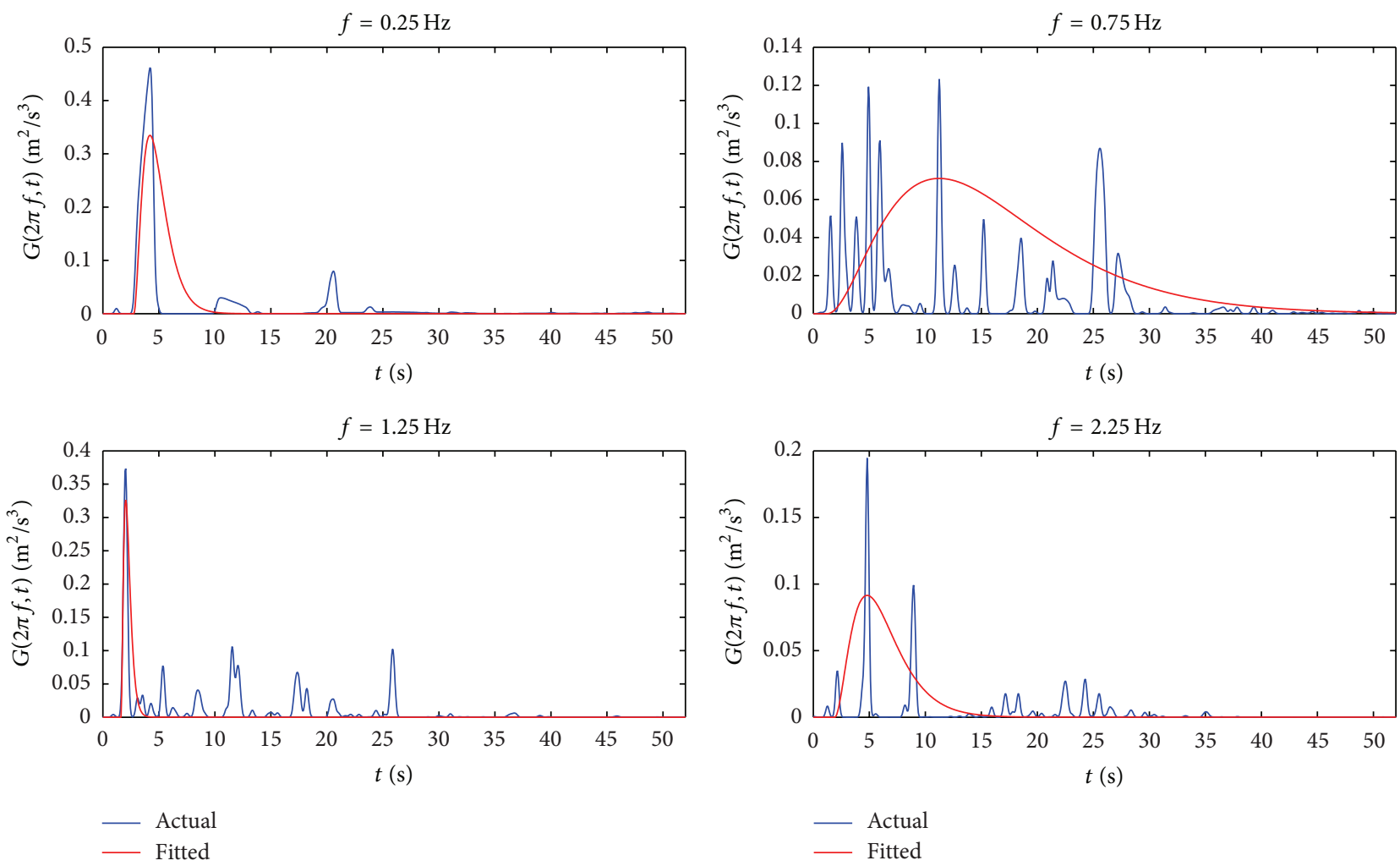

FIGURE 5: Time-varying power spectra for different frequencies of the El Centro ground motion.

where

$$
\begin{aligned}
& B_{0}(f)=-0.657+1.637 \log f-1.642(\log f)^{2} \\
& B_{1}(f)=0.563-0.208 \log f+0.0198(\log f)^{2} \\
& B_{2}(f)=1.335-0.115 \log f-0.443(\log f)^{2} \\
& P_{0}(f)=-0.808-0.929 \log f \\
& P_{1}(f)=0.123+0.134 \log f \\
& P_{2}(f)=0.357-0.083 \log f \\
& S_{0}(f)=0.0 \\
& S_{1}(f)=\left(0.863-0.509 \log f-1.141(\log f)^{2}\right) \times 10^{-2},
\end{aligned}
$$

where $t_{m}$ is the mean value of $t_{s}(f)$ at all discrete frequency points.

The attenuation law shows that the model parameters vary with the magnitude and epicentral distance of the earthquake. Using this relationship, we can calculate different time-varying power spectra according to our needs. Figure 6 shows the calculated time-varying power spectra for the same magnitude earthquake at different epicentral distances, where $t_{m}=2 \mathrm{~s}$. The power spectrum is time dependent, and its high-frequency component attenuates rapidly at long epicentral distances, which is consistent with the propagation law of ground motion.
The attenuation law shown in (14) and (15) was obtained from numerous statistical analyses of actual ground motion records. The coefficients in the equations rely on the set of ground motion records; they are not associated with the attenuation law of any other set of ground motion records. Thus, in engineering applications, it is essential to ensure the similarity of the ground motion of the site and the set of ground motions that will be statistically analyzed.

\section{Generating a Fully Nonstationary Spectrum That Is Compatible with Spatially Variable Ground Motion}

Sections 2 and 3 indicate that the new method can overcome the shortcomings of traditional simulation methods that result from the use of the simplified uniform modulation function regardless of whether the Kameda model is established based on a given ground motion or the statistical attenuation law. This paper proposes a simulation method based on the Kameda time-varying power spectrum and the evolutionary method to simulate fully nonstationary spatially variable ground motion. The core aspect of this method is replacing the traditional power spectrum model with the Kameda time-varying power spectrum model. This method can simulate ground motion with given nonstationary timefrequency characteristics. However, considering the spatial propagation characteristics of spatially variable ground motion and the engineering applications of simulated ground 


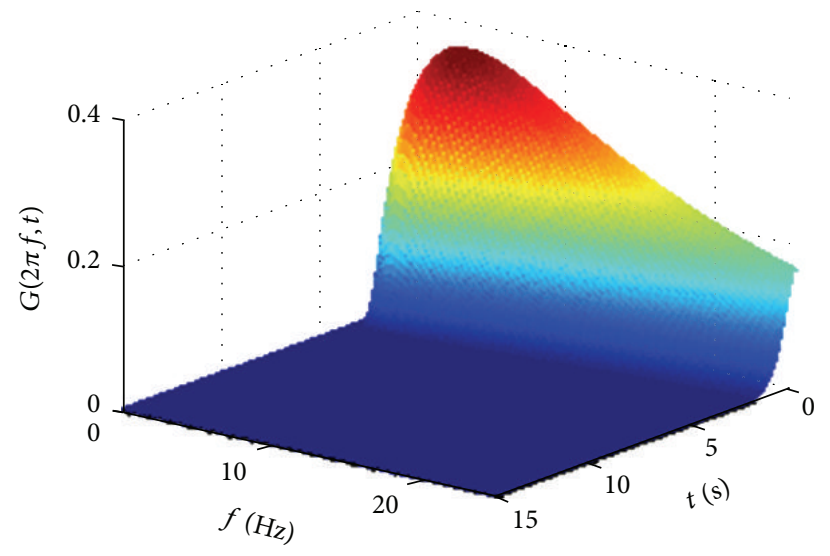

(a) $M=6.5, R=20 \mathrm{~km}$

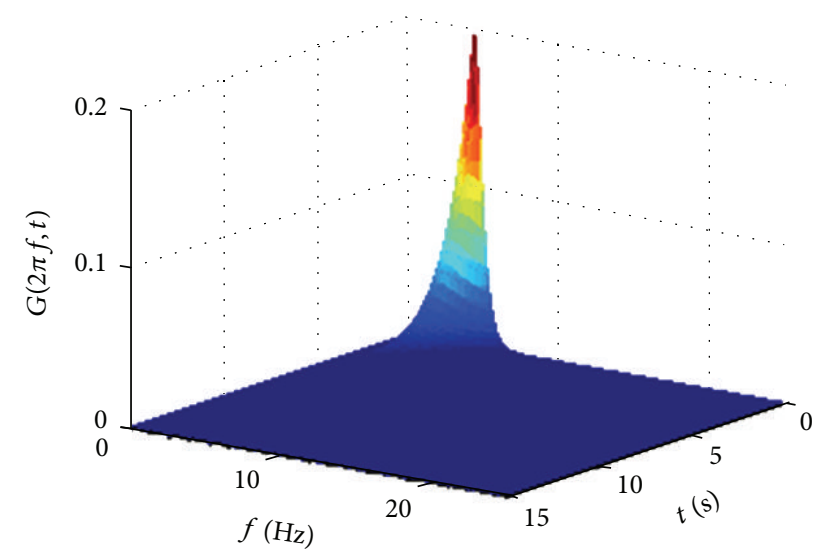

(b) $M=6.5, R=100 \mathrm{~km}$

FIGURE 6: Simulated time-varying power spectra under different conditions.

motion, two other aspects should be considered when using the Kameda model in addition to meeting the characteristics of the given time-varying power spectrum: fitting the target response spectra and the influence of the spatial characteristics. Therefore, the coherence function and response spectra-fitting technique should be included in the simulation scheme.

Figure 7 shows a schematic of a nonstationary spatially variable ground motion simulation that is based on the Kameda time-varying power spectrum model and response spectra-fitting technique. The main computing steps are as follows.

(1) Input the initial data. The main tasks in this step are defining the initial nonstationary time-frequency characteristics of the ground motion, acquiring the target response spectra and confirming the spatial effect between each point.

Two methods are available for defining the initial nonstationary time-frequency characteristics of the ground motion based on the ground motion to be simulated. The first method is based on the nonstationary time-frequency characteristics of a known ground motion and requires an actual ground motion record. The parameters of the Kameda model that match the nonstationary time-frequency characteristics of the known ground motion can be obtained using the target function shown in (13). The other method is based on the attenuation law of ground motion, which is useful when no actual ground motion is available. The initial nonstationary time-frequency characteristics rely on the attenuation law of the parameters of the Kameda model. These attenuation laws, such as the Sugito et al. model [11], can be obtained using many statistical analysis techniques.

To obtain the target response spectra when the initial ground motion is available, the response spectra of the given ground motion can be set as the target response spectra. When no ground motion is available, the target response can be obtained from the seismic design code based on the site conditions and seismic fortification. Thus, the target response spectra are the design response spectra for the seismic fortification.
The spatial effect of the ground motion at each spatial point is demonstrated by the complex correlation function, which considers the coherent effect and wave-passage effect. The function is represented as follows:

$$
\Gamma_{j k}(\omega)=\gamma_{j k}(\omega) e^{-i \omega d_{j k} / v},
$$

where $\gamma_{j k}$ and $d_{j k}$ are the coherency function and the distance between $y_{j}(t)$ and $y_{k}(t)$, respectively, and $v$ is the apparent wave velocity of the ground motion.

(2) Simulate the spatially variable ground motion based on the Kameda time-varying power spectrum. Select the Kameda time-varying power spectrum as the initial timevarying power spectrum to be simulated, and then, simulate the spatially variable ground motion using the nonstationary multivariable evolutionary spectrum method described in Section 2. The specific approaches are shown in (6)-(7) and (10)-(11).

(3) Fit the target response spectra; that is, correct the simulated ground motion to match the target response spectra. The iterative algorithm is used to fit the response spectra, in which the correction is mainly made in the frequency domain. The specific method is as follows.

For a given $j$ th point target response spectra $\operatorname{RSA}_{j}(\omega)$, suppose that $\operatorname{RSA}^{\left(y_{j}\right)}(\omega)$ is the response spectra of the $j$ th point of the simulated ground motion after the $i$ th computation and that $S_{j j}(\omega, t)$ is the corresponding time-varying power spectrum. If the target response spectra of the $i$ th computation $\operatorname{RSA}^{\left(y_{j}\right)}(\omega)$ cannot satisfy the required accuracy, the time-varying power spectrum of each point of the $i$ th iterative process should be corrected in the frequency domain according to

$$
S_{j j}^{i+1}(\omega, t)=S_{j j}^{i}(\omega, t)\left[\frac{\operatorname{RSA}_{j}(\omega)}{\operatorname{RSA}^{\left(y_{j}\right)}(\omega)}\right]^{2}, \quad j=1,2, \ldots, n .
$$

Then, recalculate the ground motion at each point using the same simulation method and repeat the procedure until the error between the response spectra of the simulated 


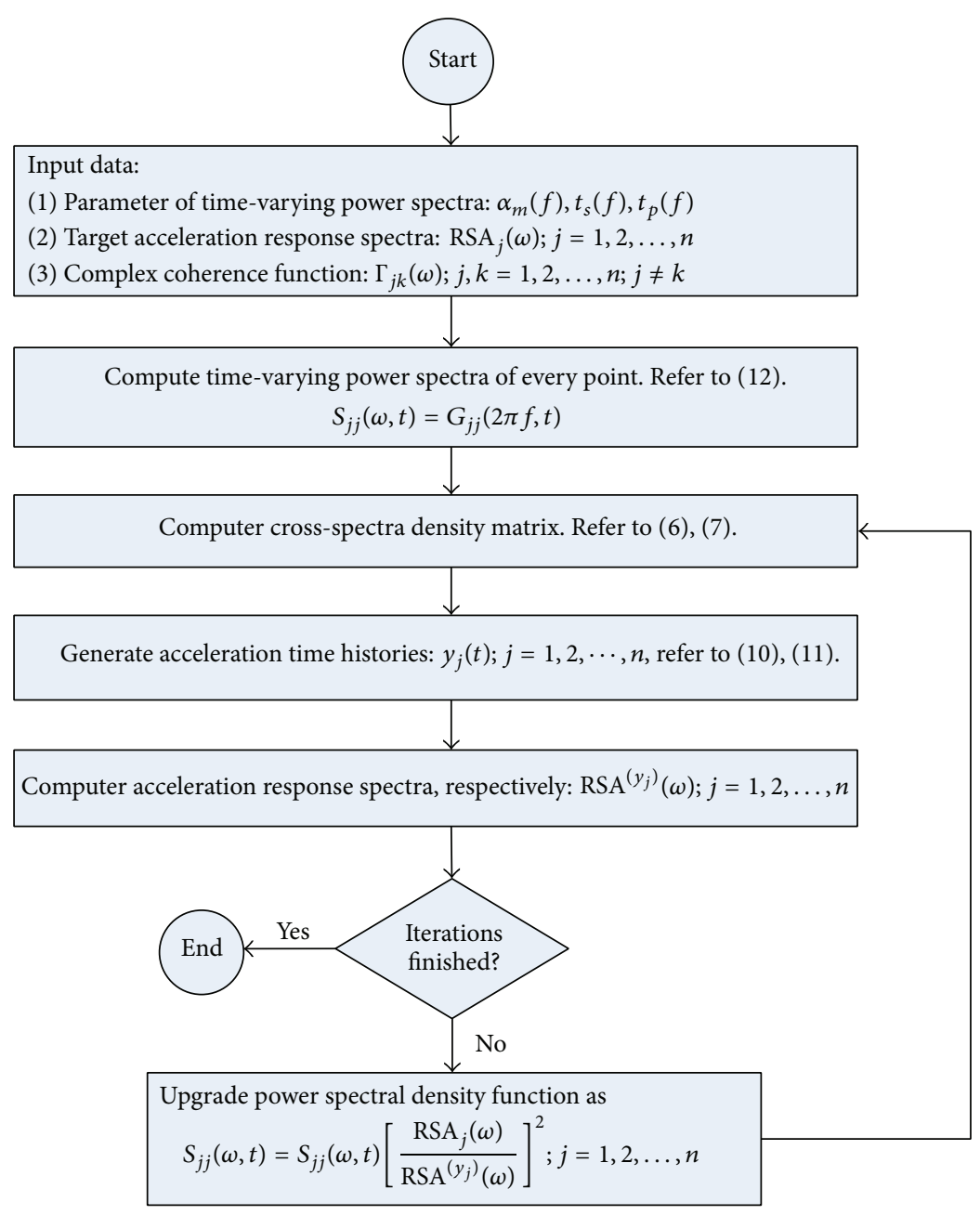

FIGURE 7: Iterative scheme to simulate the response spectrum that is compatible with nonstationary accelerations based on the time-varying power spectrum model.

ground motion and the target response spectra satisfies the required accuracy. At this point, the calculation ends.

\section{Numerical Examples}

To evaluate the ability of the proposed method to simulate nonstationary time-frequency spatially variable ground motions, two numerical examples are given in which the Kameda time-varying power spectra are obtained under two different conditions. In the first case, the parameters of the Kameda time-varying power spectrum model are identified from a given ground motion. The first example focuses on the capability of fitting a given response spectrum and its nonstationary time-frequency characteristics. In the other case, in which a ground motion is not available, the Kameda timevarying power spectrum is mainly derived from the attenuation laws of the parameters. The aim of the second example is to verify the ability of the nonstationary spatially variable ground motion to fit the response spectra of the design code.

The configuration of 3 simulated site points is shown in Figure 8 , where the arrow indicates the direction of wave propagation. In the two numerical examples, we assume that

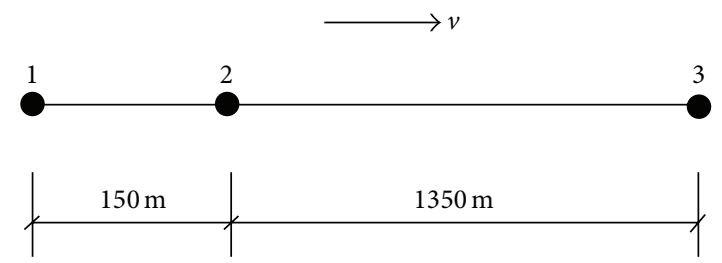

FIGURE 8: Configuration of points 1, 2, and 3 on the simulated ground site.

all of the points have the same site conditions in which the apparent wave velocity $v=1000 \mathrm{~m} / \mathrm{s}$. The HarichandranVanmarcke model [19] is selected as the coherency function model of the spatially variable ground motion, as shown in

$$
\begin{aligned}
|\gamma(\omega, d)|= & A \exp \left[-\frac{2|d|(1-A+a A)}{(a \theta(\omega))}\right] \\
& +(1-A) \exp \left[-\frac{2|d|(1-A+a A)}{\theta(\omega)}\right],
\end{aligned}
$$



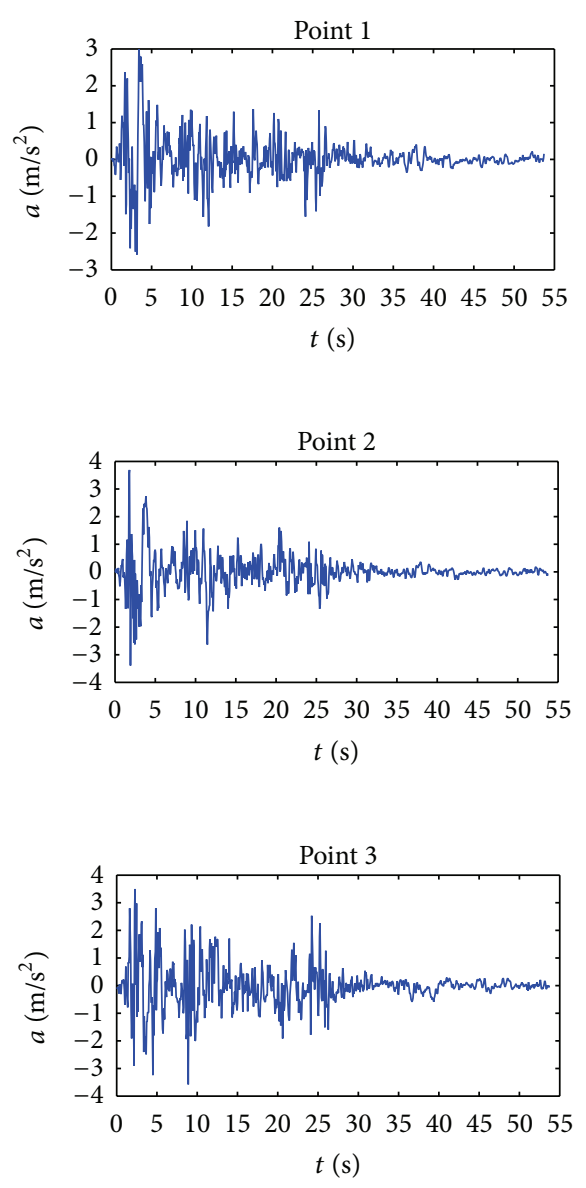

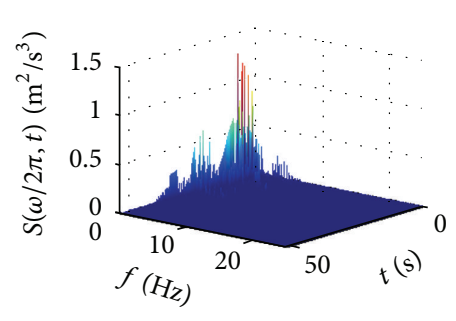

(a) Point 1
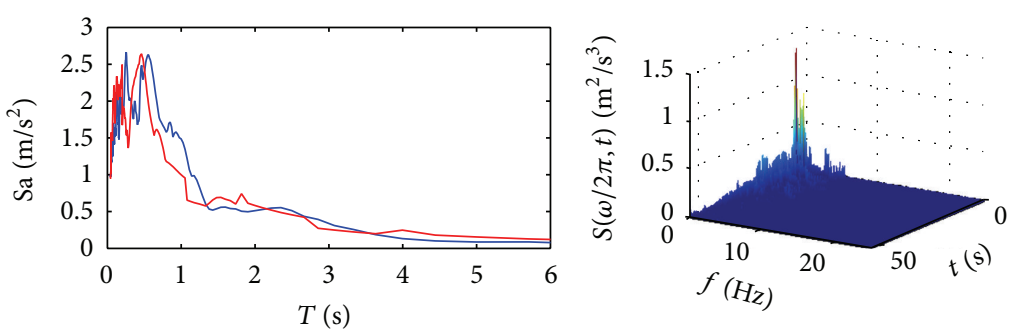

(b) Point 2
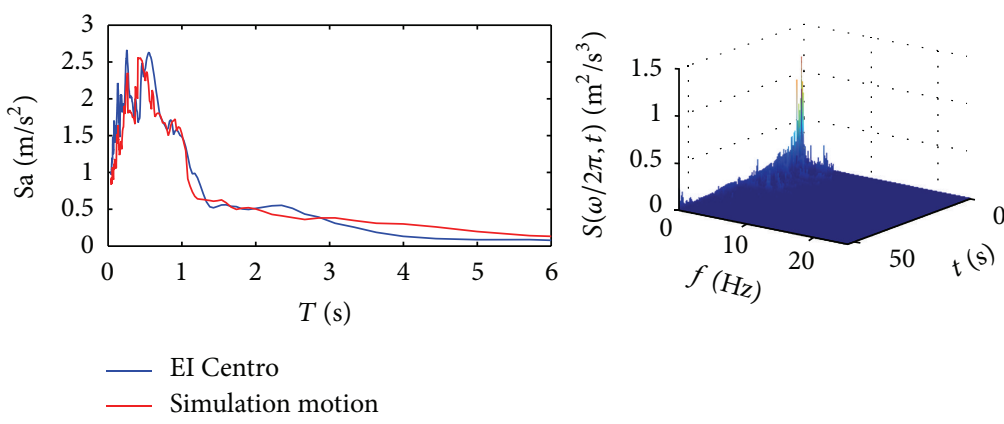

(c) Point 3

FIGURE 9: Simulated ground motions, response spectra, and time-varying power spectra for each point.

where $\theta(\omega)=k\left[1+\left(\omega / \omega_{0}\right)^{b}\right]^{-1 / 2}, A=0.736, a=0.147, k=$ $5210, \omega_{0}=6.85$, and $b=2.78$.

Condition 1. The El Centro ground motion (Figure 2(a)) is selected as the given ground motion. The simulation must match the nonstationary time-frequency characteristics and satisfy the response spectra of the given ground motion.

Figure 9 shows the simulated acceleration histories of each point as well as comparisons with the response spectra of the given ground motion and the time-varying power spectra based on the identified parameters of the Kameda time-varying power spectrum (Figure 3 ) and the iterative algorithm shown in Figure 7. The simulated ground motions fit the given response spectra well, and the nonstationary time-frequency characteristics also agree with the given ground motion, which demonstrates the effectiveness of the proposed method.

Condition 2. In the absence of an available ground motion, the Kameda time-varying power spectrum is obtained from the attenuation law. The simulation must satisfy the response spectra of the design code.

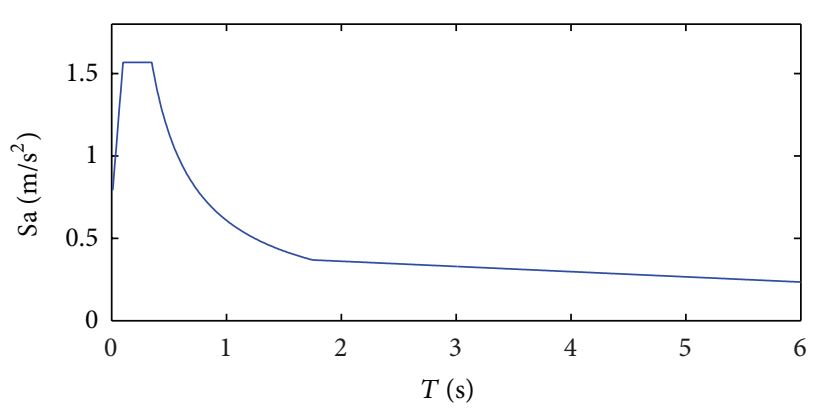

Figure 10: Design response spectra.

Based on the attenuation law of the Kameda model suggested by Sugito, we can simulate the design response spectra of the Chinese seismic design code, which is shown in Figure 10. Without loss of generality, the magnitude, epicentral distance, seismic intensity, and site classification are assumed as 7, $100 \mathrm{~km}, 8$ degrees, and Class II, respectively.

Based on the given conditions, the Kameda time-varying power spectrum can be calculated from the attenuation law 

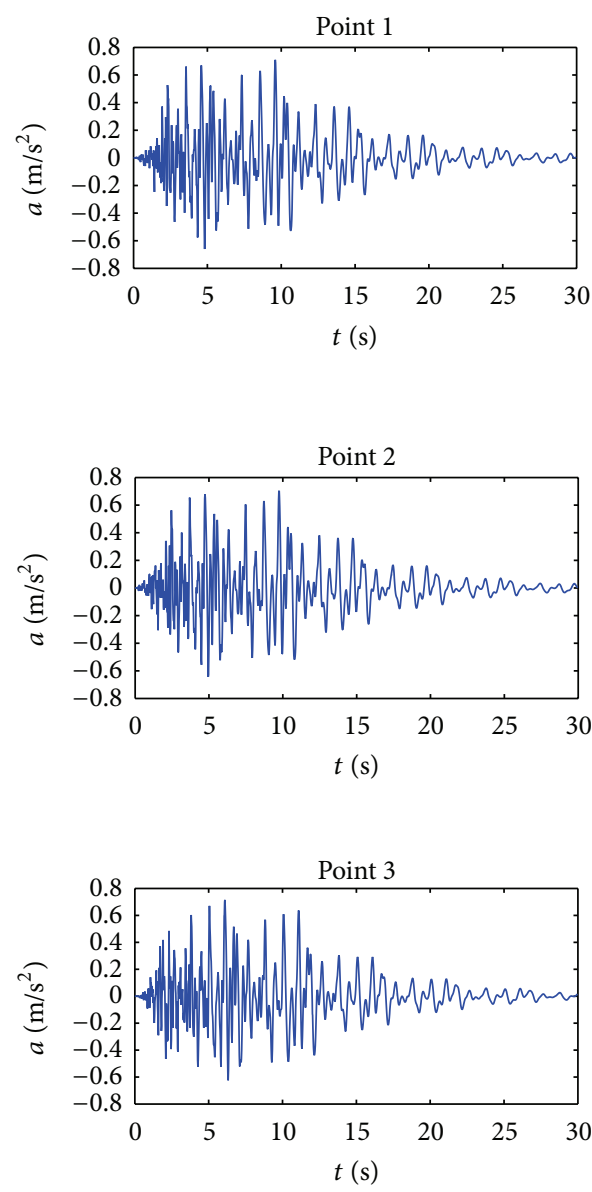

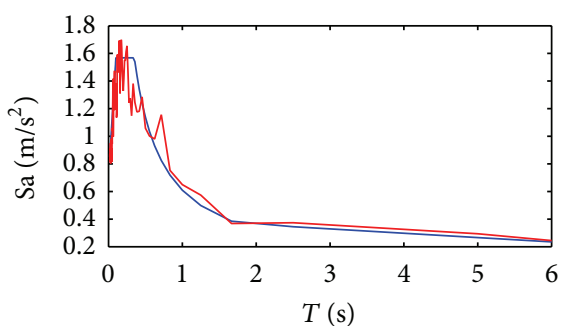

(a) Point 1

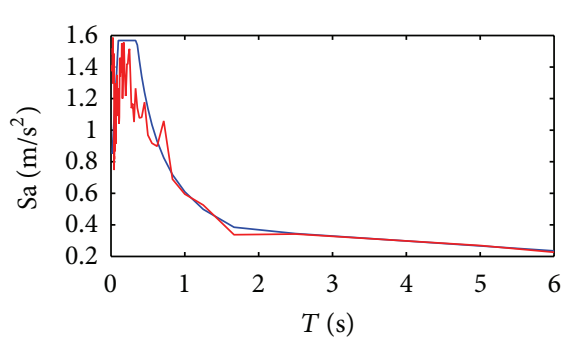

(b) Point 2

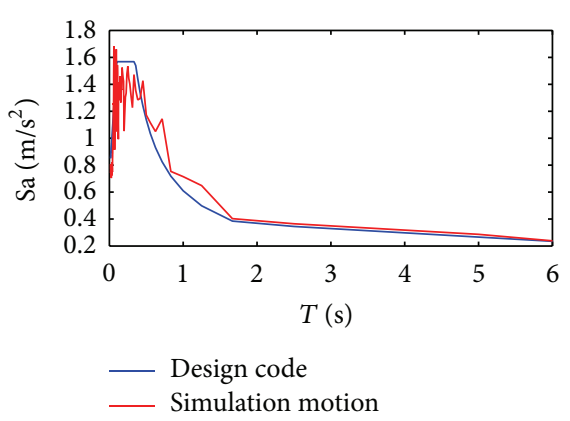

(c) Point 3
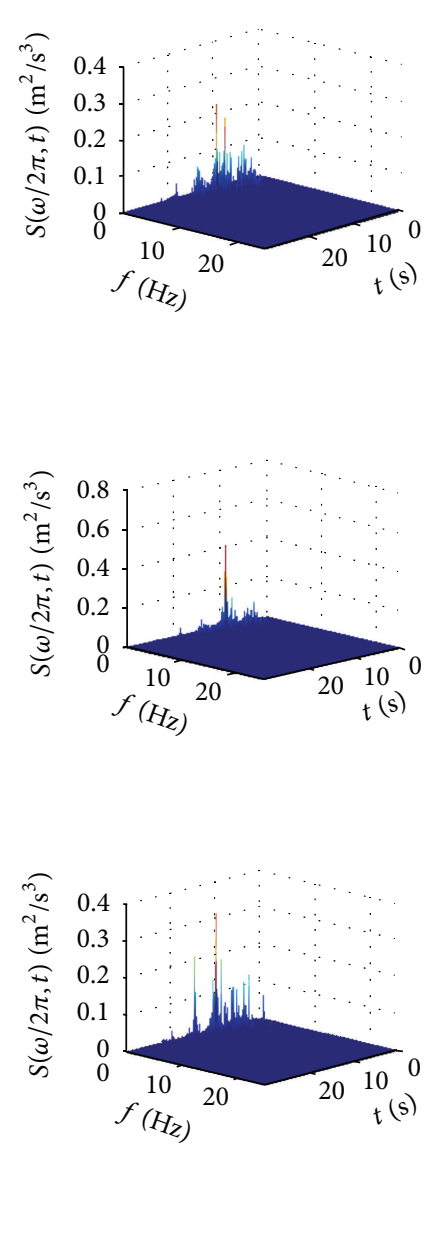

FIGURE 11: Simulated ground motions, response spectra and time-varying power spectra for each point.

represented by (14) and (15). We can obtain the simulated acceleration histories and response spectra (Figure 11) using the iterative algorithm shown in Figure 7. The attenuation law derived from the statistical analysis of the Kameda model can be used to simulate nonstationary spatially variable ground motion. After the iterative corrections, the simulation fits the response spectra of the design code well and demonstrates nonstationary characteristics in both the time and the frequency domains.

The two examples presented above demonstrate that we can simulate the fully nonstationary spatially variable ground motion with given nonstationary time-frequency characteristics using the Kameda time-varying power spectrum and simulation method for spatially variable nonstationary stochastic processes. In addition, combined with the response spectrum fitting technique, the simulation can match a target response spectrum or the response spectra of the design code. This method has many potential applications in seismic design for large-span engineering.

\section{Conclusions}

Using the evolutionary spectrum method to simulate spatially variable ground motions, this paper analyzed the shortcomings of the simplified time-varying power spectrum model used in traditional methods, which have difficulties in simulating nonstationary characteristics in the frequency domain. By introducing the Kameda time-varying power spectrum model, this paper proposes a method for simulating fully nonstationary spatially variable ground motion based on the time-varying power spectrum model. To apply the Kameda time-varying power spectrum model to the traditional evolutionary spectrum method, two examples are analyzed in which the Kameda time-varying power spectra are obtained under different conditions. A nonstationary spatially variable ground motion simulation method is proposed to fit the target response spectra based on the requirements of engineering applications.

The two numerical examples described above are based on cases in which a ground motion record is available or is 
not available. The analyses showed that the simulation based on a given ground motion can match the nonstationary timefrequency characteristics of the actual ground motion and the response spectrum. In the case where ground motion is not available, the simulation can be performed by fitting the response spectra of the design code and the Kameda timevarying power spectrum attenuation law, which is obtained through statistical analysis. The analyses of the nonstationary spatially variable ground motion simulations demonstrate the capability of the proposed method, which can be applied to analyze the dynamic seismic response of extended structures, such as long bridges, tunnels, and life-line systems.

\section{Conflict of Interests}

The authors declare that there is no conflict of interests regarding the publication of this paper.

\section{Acknowledgment}

This work was supported by National Natural Science Foundation of China under Grant no. 90815011.

\section{References}

[1] M. B. Priestley, "Power spectral analysis of non-stationary random processes," Journal of Sound and Vibration, vol. 6, no. 1, pp. 86-97, 1967.

[2] G. Deodatis, "Non-stationary stochastic vector processes: seismic ground motion applications," Probabilistic Engineering Mechanics, vol. 11, no. 3, pp. 149-168, 1996.

[3] C.-C. Tsai and Y. M. A. Hashash, "Evaluation of two approaches to simulate spatially variable ground motions," Journal of Earthquake Engineering, vol. 14, no. 2, pp. 293-308, 2010.

[4] M. Shinozuka, "Digital simulation of random processes and its applications," Journal of Sound and Vibration, vol. 25, no. 1, pp. 111-128, 1972.

[5] G. Deodatis, "Simulation of ergodic multivariate stochastic processes," Journal of Engineering Mechanics, vol. 122, no. 8, pp. 778-787, 1996.

[6] J. P. Conte and B. F. Peng, "Fully nonstationary analytical earthquake ground-motion model," Journal of Engineering Mechanics, vol. 123, no. 1, pp. 15-24, 1997.

[7] J.-N. Yang, "Simulation of random envelope processes," Journal of Sound and Vibration, vol. 21, no. 1, pp. 73-85, 1972.

[8] M. Grigoriu, "On the spectral representation method in simulation," Probabilistic Engineering Mechanics, vol. 8, no. 2, pp. 75-90, 1993.

[9] T. D. Ancheta, Engineering characterization of spatially variable earthquake ground motion [doctoral dissertation], University of California, 2010.

[10] H. Kameda, "Evolutionary spectrum of seismogram by multifilter," Journal of the Engineering Mechanics Division, vol. 101, no. 6, pp. 787-801, 1975.

[11] M. Sugito, Y. Furumoto, and T. Sugiyama, "Strong motion prediction on rock surface by superposed evolutionary spectrum," in Proceedings of the 12th World Conference on Earthquake Engineering, Auckland, New Zealand, February 2000.
[12] K. Kanai, "Semi-empirical formula for the seismic characteristics of the ground," Bulletin of the Earthquake Research Institute, University of Tokyo, vol. 35, pp. 309-324, 1957.

[13] H. Tajimi, "A statistical model of determining the maximum response of a structure during an earthquake," in Proceedings of the 2nd World Conference on Earthquake Engineering, TokyoKyoto, Japan, 1960.

[14] R. W. Clough and J. Penzien, Dynamics of Structures, McGrawHill, New York, NY, USA, 1993.

[15] M. Shinozuka and Y. Sato, "Simulation of non-stationary random process," Journal of Engineering Mechanics, no. 93, pp. 1140, 1967.

[16] M. Amin and A. H. S. Ang, "Non-stationary stochastic model of earthquake motion," Journal of Engineering Mechanics, no. 94, pp. 559-583, 1968.

[17] N. E. Huang, Z. Shen, S. R. Long et al., "The empirical mode decomposition and the Hilbert spectrum for nonlinear and non-stationary time series analysis," The Royal Society of London A, vol. 454, no. 1971, pp. 903-995, 1998.

[18] J. B. Berrill and T. C. Hanks, "High frequency amplitude errors in digitized strong motion accelerograms," Analysis of Strong Motion Earthquake Accelerograms, vol. 4, pp. 74-104, 1974.

[19] R. S. Harichandran and E. H. Vanmarcke, "Stochastic variation of earthquake ground motion in space and time," Journal of Engineering Mechanics, vol. 112, no. 2, pp. 154-174, 1986. 


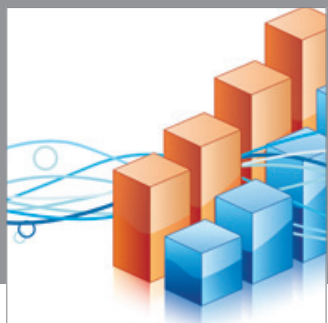

Advances in

Operations Research

mansans

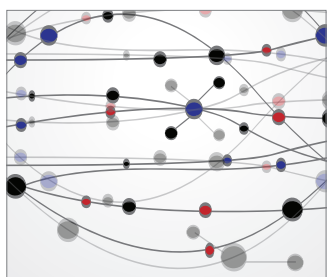

The Scientific World Journal
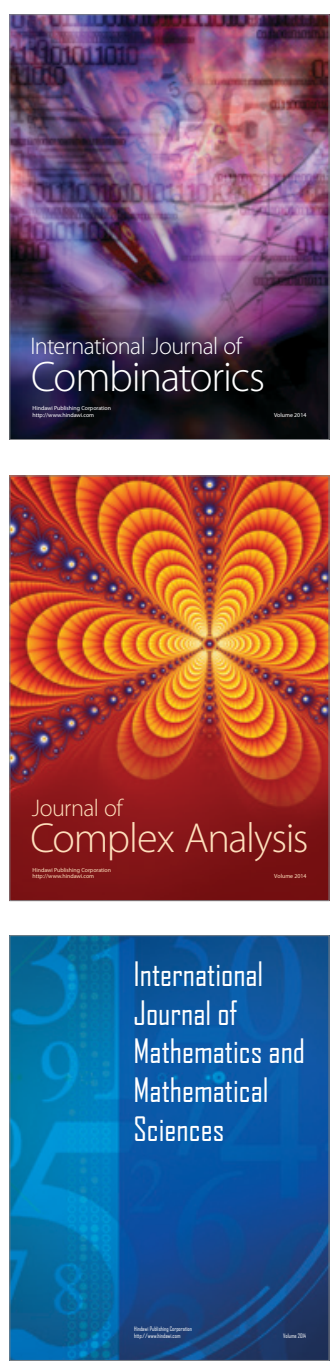
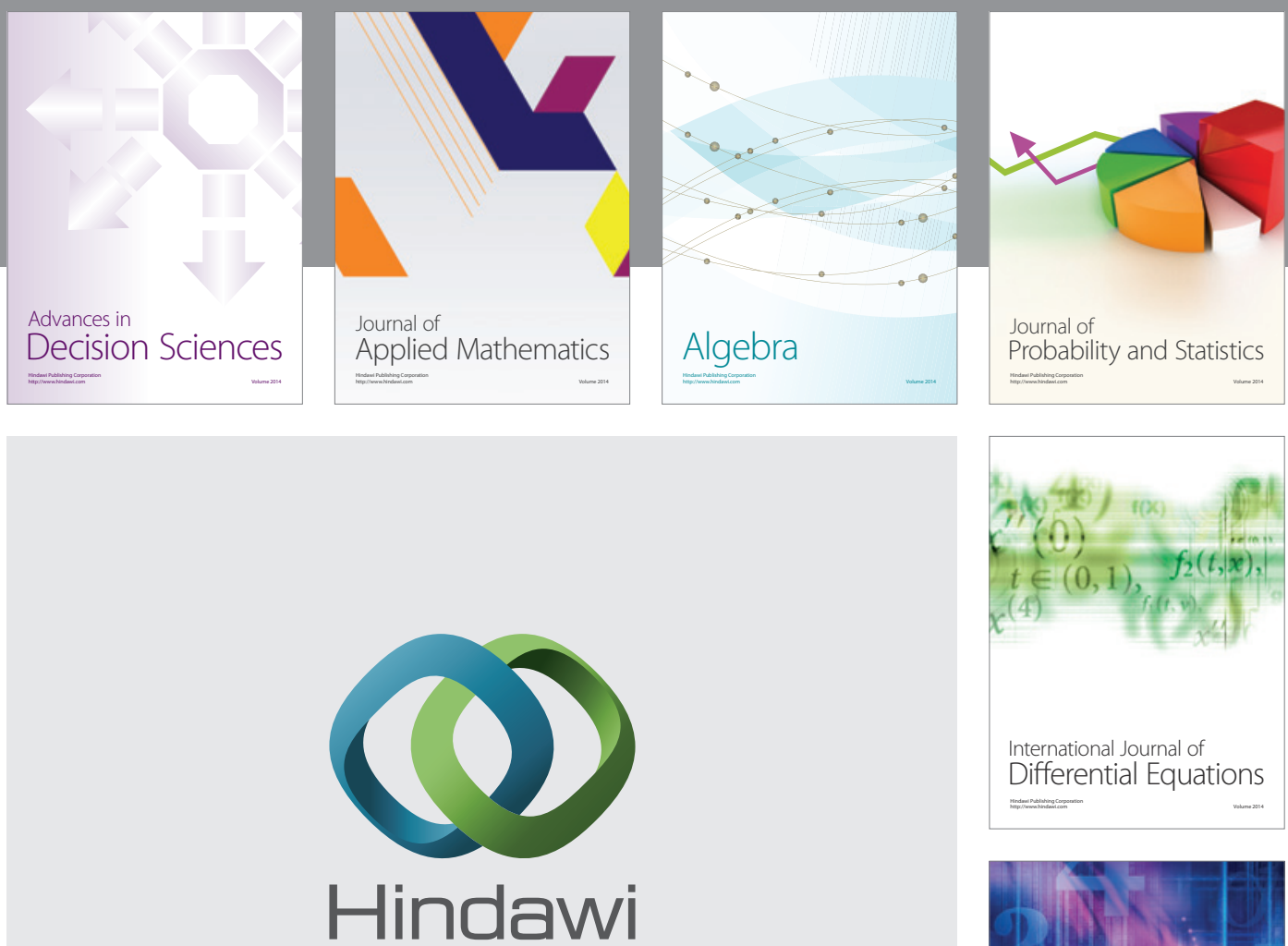

Submit your manuscripts at http://www.hindawi.com
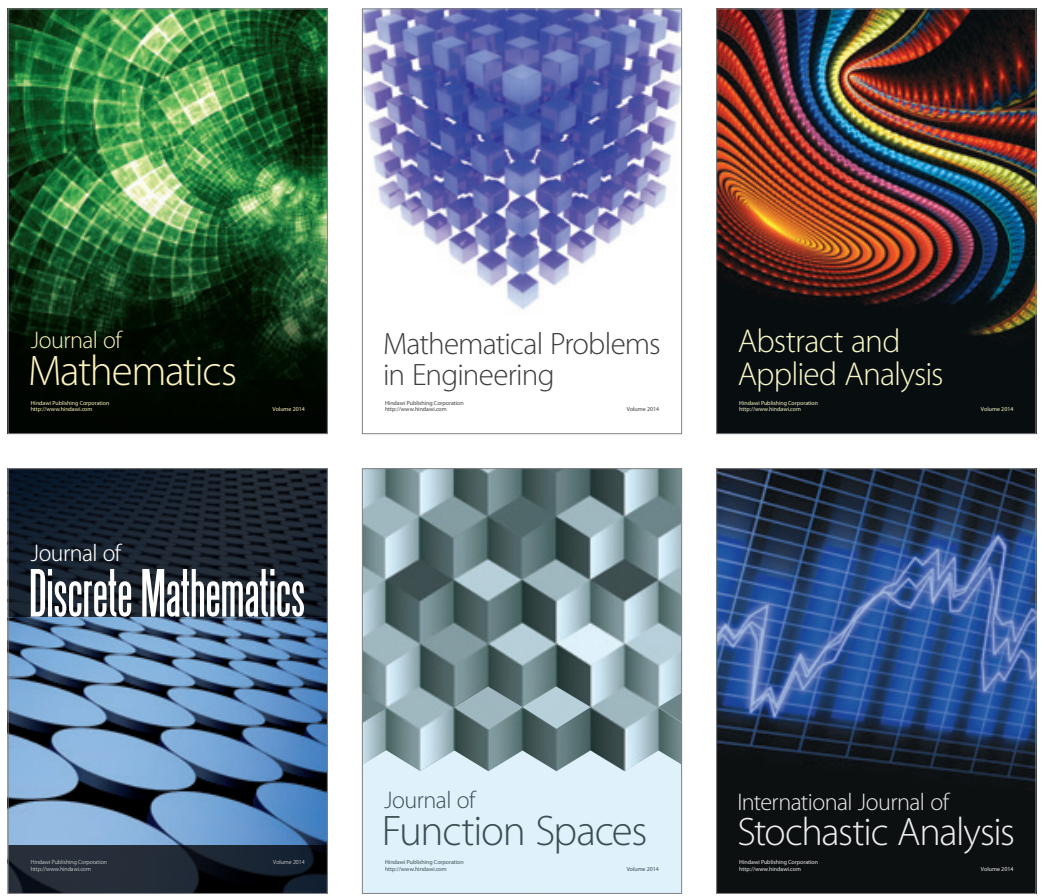

Journal of

Function Spaces

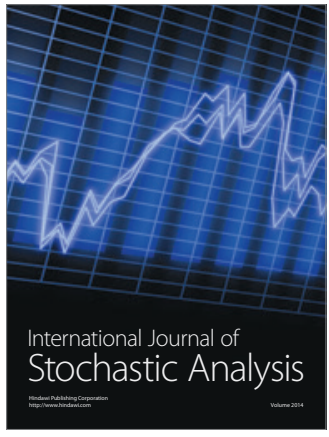

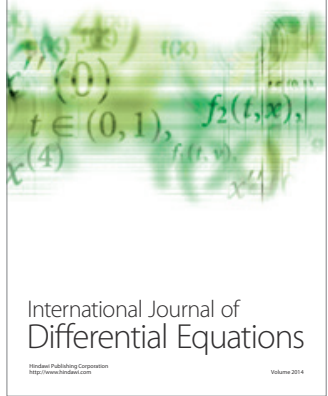
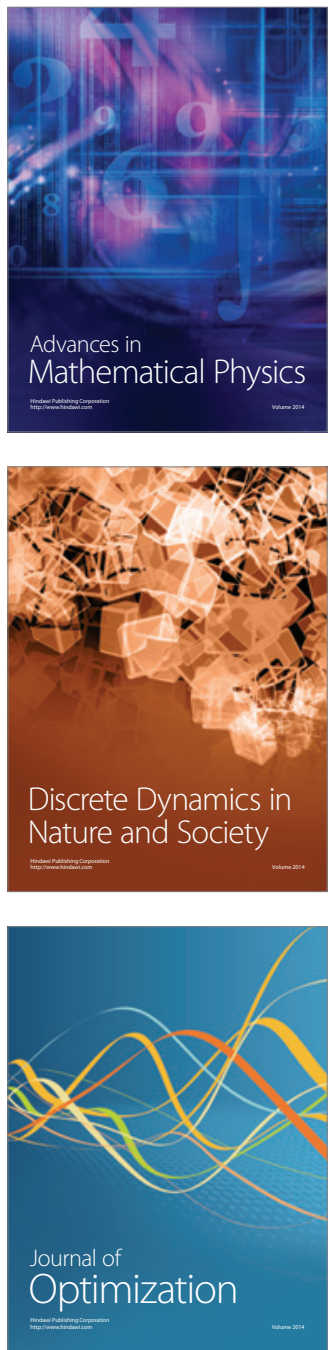